\title{
Estructura espacial y temporal de la ictiofauna del Estero de Tamarindo, Parque Nacional Marino Las Baulas, Costa Rica
}

\author{
Scarleth Ortiz-Araya ${ }^{1}$, Jonathan Chacón-Guzmán², Rigoberto Víquez-Portugués ${ }^{3}$ \\ \& Jeffrey A. Sibaja-Cordero ${ }^{4}$ \\ 1. Programa de Ciencias Marino Costeras, Universidad Nacional, Heredia, Costa Rica; scarleth.ortiz@gmail.com \\ 2. Programa Parque Marino del Pacífico, Escuela de Ciencias Biológicas, Universidad Nacional, Puntarenas, Costa Rica; \\ jonathan.chacon.guzman@una.cr \\ 3. Escuela de Ciencias Biológicas, Universidad Nacional, Heredia, Costa Rica; rviquezp@gmail.com \\ 4. Centro de Investigación en Ciencias del Mar y Limnología, Universidad de Costa Rica, San José; \\ jeffro@costarricense.cr
}

\author{
Recibido 08-III-2017. Corregido 05-IX-2017. Aceptado 03-I-2018.
}

\begin{abstract}
Spatial and temporal structure of the ichthyofauna of the Estuary of Tamarindo, Las Baulas National Marine Park, Costa Rica. Fish communities in mangrove areas have an ecological and socio-economic importance. The management in this kind of habitat is based on the data information about spatial and temporal patterns. The main goal of the study is determinate the spatial and temporal structure of the ichthic community from Tamarindo mangrove area. Six samplings trips were conducted from August 2009 to June 2010, at five mangrove stations using four different fishing gear. Also, environmental variates were recording (temperature, oxygen, salinity, $\mathrm{pH}$, and water transparency). A total of 1357 individuals belonging to 59 species of 28 families were identified. Carangidae and Lutjanidae were more richness families, and the most abundant were Poecilidae, Mugilidae and Clupeidae. Poeciliopsis elongata was the most abundant species. Temporally, the diversity reached a minimum of 1.55 in December and a maximum of 1.96 in April. The richness presented a minimum of 22 species in February and a maximum of 25 in December. Spatially, the diversity reached a minimum of 1.96 in the mouth of the estuary and a maximum of 2.79 in mid estuary; the richness a minimum of 24 species inner the estuary and a maximum of 30 at mid estuary. Additionally, a temporal pattern of succession of species was determined. The fish community shows change in the similarity in their species composition from the mouth to the inner part of the estuary. At inner of the estuary the abundance was low, in contrast with the richness, diversity and equitability. Finally, nursery process for some key species for fisheries occurs in this estuary; made it fragile and susceptible to pollution and anthropogenic impacts, because their short mangrove area and shallow depth. Rev. Biol. Trop. 66(Suppl. 1): S255-S268. Epub 2018 April 01.
\end{abstract}

Key words: Diversity indices, abundance, physicochemical conditions, spatio-temporal distribution, ichthiofaunal structure, estuary.

Los estuarios son reconocidos como los sistemas naturales más productivos del mundo, donde habitan gran número de especies propias de dicho medio; debido a los refugios y alimento que encuentran; pero a la vez, son zonas muy susceptibles debido a la descarga de efluentes industriales y urbanos que normalmente se sitúan en los márgenes de estos ecosistemas (López, 2005; De Marco, Pan, Bassini, \& Vallina, 2013; Martínez, 2015). Dentro del ecosistema de manglar, la ictiofauna constituye una de las comunidades más importantes, este grupo es propenso a ser afectado por una serie de actividades antrópicas. Recientes estudios se han enfocado en investigar sobre la composición y estructura espacio-temporal y así optar por medidas de manejo que la proteja (Benítez et al., 2007; Sandoval-Huerta, Madrigal-Guridi, Escalera-Vázquez, Medina-Nava, \& Domínguez-Domínguez, 2014; Carrasco \& 
Soriguer, 2015). Para la protección de estas comunidades se utiliza el conocimiento de su interacción con un gran número de factores y procesos ambientales que causan diferencias de una ubicación a otra e igualmente los cambios temporales, influencia de variables ambientales propias de cada época climática (Jiménez, 1999; Bejarano, Blanco, \& Mojica, 2006).

Existen varios estudios sobre la dinámica y estructura de comunidades de peces en estuarios y manglares de climas tropicales; en particular, el Pacífico costarricense ha sido beneficiado con múltiples investigaciones en arroyos, pozas intermareales y otras cuencas hidrográficas (Alpírez, 1985; Burcham, 1988; Bussing \& López, 1977; Chicas; 2001). Se cuenta con evaluaciones de la composición y estructura de varias comunidades ícticas (Constanz, Bussing, \& Saul, 1981; Winemiller, 1983; Phillips, Astorga, Hidalgo, \& Villarreal, 1983; Winemiller \& Morales, 1989; Rojas, Pizarro, \& Castro, 1994; Araya, 1998; Espinoza, 2007) y estudios con listas de especies y algunos datos ecológicos en ambientes acuáticos (Rojas et al., 1994; Chicas, 2001; Tiffer et al., 2003; Feutry, Hartmann, Casabonnet, \& Umaña, 2010).

Un estuario amenazado en Costa Rica, especialmente por una creciente urbanización en sus márgenes, es el estero de Tamarindo que es un humedal de importancia Internacional Ramsar, desde 1993. Este es caracterizado por ser un sitio crianza de especies de importancia pesquera, donde a pesar de ello, solamente se han realizado listas de especies con información zoogeográfica (Tiffer et al., 2003). Un conocimiento ecológico de la dinámica de la comunidad de peces, tomando en cuenta su abundancia y frecuencia, permite determinar cuáles especies y en qué zonas están presentes, a lo largo del año. Además, conocer cómo estas especies se encuentran relacionadas a las condiciones físico químicas del agua, la marea, descargas de ríos, topográfia y vegetación de cada sitio; respuestas que pueden asociarse a potenciales factores antropogénicos que podrían afectarlos y determinar medidas de manejo y mitigación. Así, con el propósito de apoyar los esfuerzos de manejo, conservación y uso sostenible de la ictiofauna del estero de Tamarindo, este trabajo relaciona la composición de la comunidad íctica con patrones espacio temporales influenciados por las variables ambientales.

\section{MATERIALES Y MÉTODOS}

La investigación se realizó en el estero de Tamarindo, Parque Nacional Marino las Baulas $\left(10^{\circ} 18^{\prime} 53^{\prime \prime} \mathrm{N}-85^{\circ} 50^{\prime} 2.2{ }^{\prime} \mathrm{W}\right)$, Guanacaste, Costa Rica. Este ecosistema es influenciado por los ríos Matapalo, San Andrés, Quebrada Honda y Quebrada Barco (Tiffer et al., 2003). Los muestreos se llevaron a cabo en agosto, octubre y diciembre del 2009, y febrero, abril y junio del 2010 (tres muestreos en estación seca y tres en la lluviosa). El trabajo de campo consideró variables ambientales como la profundidad, vegetación, tipo de fondo, corrientes e influencia de los ríos. Para la recolecta de los peces se seleccionaron cinco estaciones distribuidas desde la boca a los dos principales ramales internos del estero (Fig. 1, Cuadro 1).

Comportamiento de las variables físico químicas: Las variables fisicoquímicas monitoreadas en cada estación y muestreo fueron la profundidad media, obtenida por medio de una cuerda graduada $(\mathrm{cm})$, promediando varias medidas a lo ancho del cauce, el $\mathrm{pH}$ con un pHmetro portátil YSI, $200^{\circledR}$, la salinidad (\%) con un refractómetro de luz YSI ${ }^{\circledR}$, y el oxígeno disuelto $(\mathrm{mg} / \mathrm{l})$ y la temperatura $\left({ }^{\circ} \mathrm{C}\right)$ con un oxigenómetro YSI $95^{\circledR}$. La transparencia del agua se midió con un disco Secchi (m/profundidad).

Para conocer si el promedio de las variables fisicoquímicas varió entre los muestreos y entre las estaciones se realizaron análisis de varianza (modelos factoriales) (Quinn \& Keough, 2002; Crawley, 2007). Se realizó un análisis de similitudes (ANOSIM) de dos vías sin réplicas con la distancia euclidiana de la matriz de datos ambientales estandarizada, para determinar el cambio entre las estaciones y los muestreos y cuál era el grado de efecto de todas las variables ambientales en dichas diferencias (valor de grado R, si la diferencia es total el 
CUADRO 1

Descripción y ubicación de las estaciones de muestreo de peces en el estero de Tamarindo

TABLE 1

Description and location of the sampling stations of fishes in the Tamarindo Estuary

\begin{tabular}{|c|c|c|c|}
\hline Estación & Ubicación & Longitud & Características \\
\hline 1 & $\begin{array}{l}\text { Boca del estero } \\
\left(10^{\circ} 18^{\prime} 27.59^{\prime \prime} \mathrm{N}-85^{\circ} 50^{\prime} 17.18^{\prime \prime} \mathrm{O}\right)\end{array}$ & $600 \mathrm{~m}$ & $\begin{array}{l}\text { Corrientes fuertes, aguas claras, poca vegetación } \\
\text { sumergida y fondos y orillas de arena. }\end{array}$ \\
\hline 2 & $\begin{array}{l}\text { Tramo principal medio } \\
\left(10^{\circ} 19^{\prime} 12.21^{\prime \prime} \mathrm{N}-85^{\circ} 50^{\prime} 4.34^{\prime \prime} \mathrm{O}\right)\end{array}$ & $1300 \mathrm{~m}$ & $\begin{array}{l}\text { Corrientes fuertes, aguas claras, orillas con vegetación de } \\
\text { manglar sumergida y fondos de arena, roca sólida y lodo. }\end{array}$ \\
\hline 3 & $\begin{array}{l}\text { Zona de convergencia } \\
\left(85^{\circ} 19^{\prime} 29.98^{\prime \prime} \mathrm{N}-85^{\circ} 49^{\prime} 54.60^{\prime \prime} \mathrm{O}\right)\end{array}$ & $250 \mathrm{~m}$ & $\begin{array}{l}\text { Corrientes de moderadas a fuertes, aguas turbias, } \\
\text { vegetación de manglar sumergida con algunos parches de } \\
\text { playa de arena y lodo, y fondos de lodo y arena. }\end{array}$ \\
\hline 4 & $\begin{array}{l}\text { Canal norte } \\
\left(10^{\circ} 19^{\prime} 52.12^{\prime \prime} \mathrm{N}-85^{\circ} 49^{\prime} 52.08^{\prime \prime} \mathrm{O}\right)\end{array}$ & $900 \mathrm{~m}$ & $\begin{array}{l}\text { Corrientes moderadas, aguas turbias, vegetación de } \\
\text { manglar sumergida, con algunos parches de playa de } \\
\text { lodo y fondos de lodo. }\end{array}$ \\
\hline 5 & $\begin{array}{l}\text { Canal este } \\
\left(10^{\circ} 19^{\prime} 26.43^{\prime \prime} \mathrm{N}-85^{\circ} 49^{\prime} 43.66^{\prime \prime} \mathrm{O}\right)\end{array}$ & $800 \mathrm{~m}$ & $\begin{array}{l}\text { Cauce sinuoso, en gran parte bajo la sombre del mangle, } \\
\text { corrientes moderadas, aguas turbias, vegetación de } \\
\text { manglar sumergida, con algunos parches de playa de } \\
\text { lodo, y fondos de lodo. }\end{array}$ \\
\hline
\end{tabular}

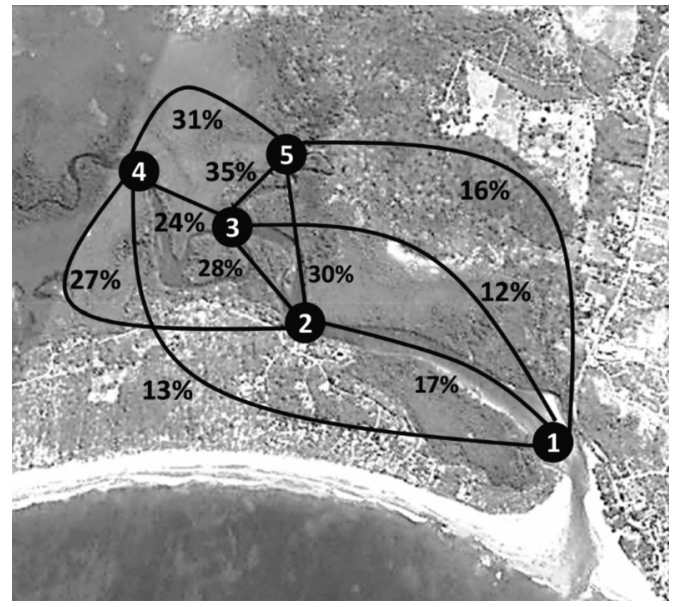

Fig. 1. Comparación porcentual (similitud de Bray-Curtis) entre la composición íctica de cada estación del estero de Tamarindo. Números del 1 a 5 son las estaciones.

Fig. 1. Percentual comparison (Bray-Curtis similarity) of the ichthyofaunal composition between stations at Tamarindo estuary. Number of 1 to 5 are the sampling stations.

valor de R el 1) (Clark \& Warcwick, 1994; Hammer, Harper, \& Ryan, 2001).

Estructura de la comunidad íctica y su variación espacio temporal: Para la recolecta de especímenes se utilizó un chinchorro playero de, $25 \mathrm{~m}$ de largo por $1.2 \mathrm{~m}$ de alto con una luz de malla de, $2.54 \mathrm{~cm}$, un trasmallo de monofilamento de $100 \mathrm{~m}$ de ancho por, $2 \mathrm{~m}$ de alto con una luz de malla de $7.72 \mathrm{~cm}$, una atarraya de, $2 \mathrm{~m}$ de diámetro con una luz de malla de $1.27 \mathrm{~cm}$, y cuerdas con anzuelos números 8 , 13 y 18 utilizando sardina cómo carnada. Para estandarizar las capturas, en cada estación de muestreo, los artes de pesca fueron utilizados en un transecto de $100 \mathrm{~m}$. Con el chinchorro se realizaron dos barridos por estación (área de barrido $500 \mathrm{~m}^{2}$ ); El trasmallo se sumergió por $60 \mathrm{~min}$; las líneas de mano fueron lanzadas reiteradamente por $60 \mathrm{~min}$ y con la atarraya se realizaron diez lances por estación. Solamente un pez por especie fue sacrificado para la identificación, la cual se realizó en los sitios de captura con las guías Bussing \& López (1994), Fischer et al. (1995), Allen \& Robertson (1998) y Nelson (2006). Los datos fueron separados por estación y por muestreo para sus respectivos análisis estadísticos.

Se calcularon los índices ecológicos de Shannon-Weiner (base ln), equitatividad, abundancia y riqueza absoluta (Krebs, 1999). Los índices se compararon mediante el software PAST (Hammer et al., 2001); por el método de bootstrap para determinar diferencias en espacio y tiempo en el estuario. Para conocer 
si había variación de estos índices entre los muestreos y entre las estaciones, se realizó un análisis de varianza de dos vías (mediciones repetidas sin réplicas) (Quinn \& Keough, 2002; Crawley, 2007).

Se determinó el cambio espacial del porcentaje de similitud de la composición de la ictiofauna dentro del estuario, graficando sobre un mapa de la región de estudio el promedio de similitud (basada en el índice de Bray Curtis) entre estaciones. Además, con la matriz de presencia ausencia de especies entre visitas se corrió una prueba de seriación en el programa PAST, para determinar si el patrón de aparición de especie fue aleatorio o se dio un cambio asociado con la fecha de muestreo (Hammer et al., 2001).

\section{Relación de la ictiofauna con las varia-} bles ambientales: Mediante análisis de correspondencias canonícas con el número de individuos por especie en cada estación de muestreo, se buscó relacionar la ictiofauna con las variables ambientales (Clark \& Warwick, 1994; Hammer et al., 2001; Quinn \& Keought, 2002). Utilizando el análisis BIOENV (método de Spearman), del programa PRIMER 6.0, se pudo conocer cuáles variables ambientales son las que mejor explican los cambios de la composición de la ictiofauna en el estuario, para lo cual se utilizó la matriz de similitud de Bray-Curtis de la ictiofauna y las variables ambientales estandarizadas.

\section{RESULTADOS}

Comportamiento de las variables físico químicas: La profundidad en cada estación fue muy variable debido a que el cauce presentó fondos irregulares, a la variación de las mareas y a que esta variable se determinó desde la orilla hasta el centro del cauce, en cinco sitios a lo largo de cada estación. El rango de profundidad entre estaciones estuvo entre $0.83 \pm 0.38 \mathrm{~m}$ en la boca hasta $1.2 \pm 0.60 \mathrm{~m}$ en la estación dos, alcanzando $4.5 \mathrm{~m}$ en las zonas de mayor profundidad con las mareas más altas del año. Sin embargo, la profundidad esta variable no presentó diferencias a lo largo del estero (F; $\mathrm{p}>0.05$ ).

La concentración de oxígeno disuelto en el agua fue mayor durante febrero y abril (6.8 $\mathrm{mg} / \mathrm{l})$, y más alta en las estaciones ubicadas cercanas a la boca $(\mathrm{F} ; \mathrm{p}<0.05)$. La temperatura registró valores bajos en octubre $\left(26.2{ }^{\circ} \mathrm{C}\right)$ y altos en febrero y abril $\left(29.8^{\circ} \mathrm{C}\right)(\mathrm{F} ; \mathrm{p}<0.05)$. No se registraron cambios entre estaciones $(\mathrm{F}$; $p>0.05)$. Las diferencias en salinidad entre estaciones fueron mayores en los periodos de lluvia, llegando en la estación cinco a 2 ppt. En la época seca las estaciones tres y cinco presentaron los valores promedio más bajos de salinidad (26 y, $24 \mathrm{ppt}$, respectivamente) (F; p $<0.05)$. El pH decreció de la boca (8.3) al interior del estero $(7.9)(\mathrm{F} ; \mathrm{p}<0.05)$. La estación uno presentó mayor transparencia $(100 \%)$ que el resto $(\mathrm{F} ; \mathrm{p}<0.05)$. Temporalmente, la transparencia fue mayor en abril $(3.6 \mathrm{~m})$, y menor en junio $(0.75 \mathrm{~m})(\mathrm{F} ; \mathrm{p}<0.05)$ (Cuadro 2). El cambio ambiental entre estaciones fue de $38 \%$ (ANOSIM; $p<0.001$ ) y entre los meses de 73 $\%$ (ANOSIM; $p<0.001$ ).

Estructura ictiofaunística y variación espacio temporal: El número total de especímenes recolectados fue de 1357 individuos, el índice general de diversidad fue de 2.68 y el de equidad fue de 0.65 . La diversidad se distribuyó en 59 especies pertenecientes a 28 familias (Cuadro 3).

El número de individuos capturados varió en el tiempo y el espacio. Fluctuó durante todo el periodo de estudio, con su punto máximo en octubre (F; $\mathrm{p}<0.05$, Cuadro 4$)$, y cambió de una estación a otra, con la mayor abundancia en la estación tres (F; p $<0.05$, Cuadro 4). Las familias Poecilidae, Mugilidae y Clupeidae fueron las más abundantes conformando un total de 799 individuos en conjunto y representando el $58.8 \%$ del total capturado. Temporalmente, la riqueza absoluta fue similar (entre 24 y 25 especies) durante todo el periodo de estudio ( $\mathrm{F} ; \mathrm{p}>0.05$, Cuadro 4), mientras las estaciones dos y tres fueron donde más especies (30) se recolectaron $(\mathrm{F} ; \mathrm{p}<0.05$, Cuadro 4) siendo mayor en la estación tres. Las 
CUADRO 2

Valores promedio de los factores fisicoquímicos determinados en cada estación y cada muestreo efectuado en el estero de Tamarindo

TABLE 2

Average values of the physicochemical factors determined in each season and each sampling carried out in the Tamarindo estuary

\begin{tabular}{|c|c|c|c|c|c|c|}
\hline Muestreo & Estación & $\mathrm{O} 2$ & ${ }^{\circ} \mathrm{C}$ & $\%$ & $\mathrm{pH}$ & Transp \\
\hline Ago & 1 & $6.4 \pm 0.1$ & $29.6 \pm 0.2$ & $34.0 \pm 0.0$ & $8.2 \pm 0.0$ & $4.6 \pm 0.5$ \\
\hline Ago & 2 & $6.5 \pm 0.1$ & $29.4 \pm 0.4$ & $34.0 \pm 0.0$ & $8.2 \pm 0.0$ & $4.9 \pm 0.5$ \\
\hline Ago & 3 & $4.0 \pm 0.2$ & $29.4 \pm 0.2$ & $34.0 \pm 0.0$ & $7.7 \pm 0.3$ & $1.6 \pm 0.6$ \\
\hline Ago & 4 & $3.4 \pm 0.2$ & $29.5 \pm 0.2$ & $34.0 \pm 0.0$ & $7.5 \pm 0.1$ & $2.3 \pm 0.4$ \\
\hline Ago & 5 & $4.1 \pm 0.8$ & $29.8 \pm 0.1$ & $32.0 \pm 0.0$ & $7.6 \pm 0.1$ & $1.2 \pm 1.1$ \\
\hline Oct & 1 & $5.6 \pm 0.1$ & $28.7 \pm 0.4$ & $29.4 \pm 1.1$ & $8.4 \pm 0.2$ & $3.1 \pm 1.0$ \\
\hline Oct & 2 & $5.5 \pm 0.1$ & $28.5 \pm 0.6$ & $29.1 \pm 1.4$ & $8.3 \pm 0.2$ & $3.1 \pm 1.1$ \\
\hline Oct & 3 & $5.0 \pm 0.1$ & $27.9 \pm 0.6$ & $21.9 \pm 2.5$ & $7.8 \pm 0.1$ & $1.2 \pm 0.9$ \\
\hline Oct & 4 & $3.8 \pm 0.1$ & $27.0 \pm 0.6$ & $25.6 \pm 0.5$ & $7.7 \pm 0.1$ & $1.1 \pm 0.8$ \\
\hline Oct & 5 & $3.9 \pm 0.1$ & $26.3 \pm 0.7$ & $9.5 \pm 0.5$ & $8.0 \pm 0.0$ & $0.8 \pm 1.0$ \\
\hline Dic & 1 & $4.8 \pm 0.3$ & $27.4 \pm 0.1$ & $32.3 \pm 1.2$ & $8.1 \pm 0.1$ & $2.4 \pm 1.2$ \\
\hline Dic & 2 & $4.2 \pm 0.3$ & $27.4 \pm 0.5$ & $30.9 \pm 0.8$ & $7.8 \pm 0.0$ & $1.3 \pm 0.8$ \\
\hline Dic & 3 & $3.7 \pm 0.7$ & $27.3 \pm 0.4$ & $26.8 \pm 2.0$ & $7.6 \pm 0.2$ & $1.0 \pm 0.8$ \\
\hline Dic & 4 & $3.3 \pm 0.7$ & $27.3 \pm 0.6$ & $33.2 \pm 0.9$ & $7.6 \pm 0.1$ & $1.4 \pm 0.7$ \\
\hline Dic & 5 & $3.4 \pm 0.5$ & $27.0 \pm 0.2$ & $21.1 \pm 4.9$ & $7.6 \pm 0.1$ & $1.0 \pm 0.6$ \\
\hline Feb & 1 & $6.8 \pm 0.1$ & $28.0 \pm 0.4$ & $34.3 \pm 0.5$ & $8.1 \pm 0.1$ & $1.6 \pm 1.8$ \\
\hline Feb & 2 & $6.9 \pm 0.0$ & $28.2 \pm 0.5$ & $33.9 \pm 1.6$ & $8.1 \pm 0.1$ & $1.9 \pm 1.5$ \\
\hline Feb & 3 & $6.5 \pm 0.1$ & $27.7 \pm 0.7$ & $34.3 \pm 1.6$ & $7.8 \pm 0.2$ & $1.7 \pm 1.6$ \\
\hline Feb & 4 & $6.4 \pm 0.0$ & $28.3 \pm 0.5$ & $33.7 \pm 2.0$ & $7.8 \pm 0.3$ & $2.3 \pm 2.0$ \\
\hline Feb & 5 & $6.0 \pm 0.4$ & $28.3 \pm 0.4$ & $34.2 \pm 1.5$ & $7.7 \pm 0.3$ & $1.7 \pm 1.3$ \\
\hline Abr & 1 & $6.9 \pm 0.1$ & $30.2 \pm 0.3$ & $34.3 \pm 1.0$ & $8.3 \pm 0.1$ & $4.9 \pm 0.8$ \\
\hline Abr & 2 & $6.8 \pm 0.0$ & $29.9 \pm 0.1$ & $32.5 \pm 1.5$ & $8.1 \pm 0.0$ & $4.9 \pm 0.7$ \\
\hline Abr & 3 & $6.8 \pm 0.1$ & $29.7 \pm 0.1$ & $31.9 \pm 0.4$ & $7.2 \pm 0.3$ & $4.4 \pm 0.9$ \\
\hline Abr & 4 & $6.3 \pm 0.1$ & $29.7 \pm 0.5$ & $34.0 \pm 1.6$ & $7.7 \pm 0.1$ & $3.5 \pm 1.8$ \\
\hline Abr & 5 & $6.0 \pm 0.1$ & $29.5 \pm 0.3$ & $28.1 \pm 1.8$ & $7.5 \pm 0.1$ & $3.9 \pm 1.7$ \\
\hline Jun & 1 & $5.7 \pm 0.3$ & $28.7 \pm 0.2$ & $25.6 \pm 6.0$ & $8.0 \pm 0.3$ & $1.6 \pm 1.7$ \\
\hline Jun & 2 & $5.3 \pm 0.1$ & $28.9 \pm 0.2$ & $14.8 \pm 9.2$ & $8.0 \pm 0.2$ & $1.0 \pm 1.3$ \\
\hline Jun & 3 & $4.0 \pm 0.5$ & $28.4 \pm 0.4$ & $11.4 \pm 5.1$ & $7.9 \pm 0.1$ & $0.6 \pm 0.8$ \\
\hline Jun & 4 & $3.8 \pm 0.8$ & $28.6 \pm 0.5$ & $10.0 \pm 6.3$ & $7.7 \pm 0.2$ & $0.8 \pm 0.9$ \\
\hline Jun & 5 & $4.5 \pm 0.7$ & $28.8 \pm 0.9$ & $15.0 \pm 12.2$ & $7.9 \pm 0.1$ & $0.7 \pm 1.2$ \\
\hline
\end{tabular}

familias con mayor riqueza fueron Carangidae y Lutjanidae con ocho y siete especies, respectivamente. Los índices de diversidad y equitatividad se mantuvieron estables $(\mathrm{F} ; \mathrm{p}>0.05)$ entre estaciones y entre los meses, no obstante, la estación dos fue la más diversa y la estación cinco fue la que se mantuvo más equitativa en el tiempo (Cuadro 4).

Al graficar la similitud promedio de composición de ictiofauna (índice de Bray Curtis) sobre la región de estudio (Fig. 1), se determinó que la estación uno solo representa un 12 y un $17 \%$ de similitud al resto de estaciones. La estación tres es la que en promedio más se asemeja con otras estaciones, especialmente con la estación cinco (35\%). La fauna cambio desde la boca al interior del estuario, donde las estaciones comparten al menos un tercio de composición.

Además, se determinó una tendencia temporal respecto a la presencia de especies en el estero de un $86 \%$ de cambio entre muestreos 
CUADRO 3

Riqueza de familias y especies de peces en el estero de Tamarindo

TABLE 3

Richness of fishes' families and species from Tamarindo estuary

\begin{tabular}{|c|c|c|c|}
\hline Familia & Género y especie & Familia & Género y especie \\
\hline Anablepidae & Oxyzygonectes dovii & Gobiidae & Ctenogobius sagittula \\
\hline \multirow[t]{4}{*}{ Atherinopsidae } & Atherinella argentea & Haemulidae & Haemulopsis elongatus \\
\hline & Atherinella eriarcha & & Haemulopsis leuciscus \\
\hline & Atherinella pachylepis & & Haemulopsis nitidus \\
\hline & Atherinella panamensis & & Pomadasys branickii \\
\hline Belonidae & Tylosurus fodiator & Hemiramphidae & Hyporhamphus unifasciatus \\
\hline \multirow[t]{8}{*}{ Carangidae } & Caranx caballus & Kyphosidae & Kyphosus elegans \\
\hline & Caranx caninus & Lutjanidae & Lutjanus aratus \\
\hline & Caranx vinctus & & Lutjanus argentiventris \\
\hline & Chloroscombrus orqueta & & Lutjanus colorado \\
\hline & Oligoplites altus & & Lutjanus guttatus \\
\hline & Oligoplites saurus & & Lutjanus jordani \\
\hline & Selene brevoortii & & Lutjanus novemfasciatus \\
\hline & Trachinotus rhodopus & & Lutjanus peru \\
\hline Carcharhinidae & Carcharhinus leucas & Mugilidae & Mugil cephalus \\
\hline \multirow[t]{5}{*}{ Centropomidae } & Centropomus armatus & & Mugil curema \\
\hline & Centropomus medius & Muraenidae & Gymnothorax dovii \\
\hline & Centropomus nigrescens & Paralichthyidae & Etropus crossotus \\
\hline & Centropomus robalito & Poeciliidae & Poeciliopsis elongata \\
\hline & Centropomus viridis & Pomacentridae & Abudefduf troschelii \\
\hline Chaetodontidae & Chaetodon humeralis & & Stegastes leucorus \\
\hline Chanidae & Chanos chanos & Scombridae & Scomberomorus sierra \\
\hline Clupeidae & Lile stolifera & Serranidae & Alphestes immaculatus \\
\hline Diodontidae & Diodon holocanthus & & Alphestes multiguttatus \\
\hline Eleotrididae & Eleotris picta & & Rypticus nigripinnis \\
\hline Elopidae & Elops affinis & Stromateidae & Peprilus ovatus \\
\hline Engraulidae & Anchoa spinifer & Tetraodontidae & Arothron meleagris \\
\hline \multirow[t]{4}{*}{ Gerreidae } & Gerres cinereus & & Sphoeroides annulatus \\
\hline & Eucinostomus currani & & \\
\hline & Eucinostomus gracilis & & \\
\hline & Diapterus peruvianus & & \\
\hline
\end{tabular}

CUADRO 4

Especies, abundancia e índices de diversidad y equidad de peces en cada estación del estero de Tamarindo

TABLE 4

Species, abundance and diversity of fishes from Tamarindo estuary

\begin{tabular}{lccccccccccc} 
& \multicolumn{1}{c}{ Estaciones } & \multicolumn{1}{c}{ Muestreos } \\
\multicolumn{1}{c}{ Estaciones } & 1 & 2 & 3 & 4 & 5 & 1 & 2 & 3 & 4 & 5 & 6 \\
Especies & 26 & 30 & 30 & 24 & 26 & 24 & 23 & 25 & 22 & 24 & 24 \\
Individuos & 196 & 164 & 670 & 135 & 193 & 183 & 351 & 242 & 211 & 239 & 131 \\
Shannon-Weiner & 1.96 & 2.79 & 1.97 & 2.72 & 2.49 & 1.79 & 1.77 & 1.55 & 1.82 & 1.96 & 1.87 \\
Equidad & 0.60 & 0.82 & 0.57 & 0.86 & 0.77 & 0.56 & 0.55 & 0.47 & 0.58 & 0.61 & 0.58 \\
\hline
\end{tabular}


( $Z=-1.32 ; \mathrm{p}=0.074$, Cuadro 5). Un grupo de seis especies solo estuvo presente al inicio del periodo de estudio, otro grupo de 14 especies solo estuvo al final del periodo de estudio, mientras que otras cinco especies estuvieron durante todo el periodo, el resto resultaron intermitentes en su aparición (Cuadro 5, Fig. 2).

Relación de la ictiofauna con las variables ambientales: En el analisis de correspondencias canonicas (Fig. 2) se puede observar como la estación uno se diferenció de las demás por presentar mayor valor de oxígeno disuelto y $\mathrm{pH}$, y por la gran presencia de Caranx caballus, Arothron meleagirs y Pomadasys nitidus. Se puede apreciar en la Fig. 2 cómo las especies que generalmente habitan aguas oceánicas se encuentran cercanas a valores de alta salinidad como es el caso del pargo manchado (Lutjanus guttatus), y el pargo negro (Lutjanus novemfasciatus) mientras que especies que pueden tolerar rangos inferiores se encuentran

\section{CUADRO 5}

Presencia y ausencia de las especies de peces a lo largo del periodo de estudios en el estero de Tamarindo. $\mathrm{P}=\mathrm{Presencia}$

TABLE 5

Presence and absence of fishes species throughout the study period in the Tamarindo Estuary. $\mathrm{P}=$ Presence

\begin{tabular}{|c|c|c|c|c|c|c|}
\hline \multirow{2}{*}{ Especie } & \multicolumn{6}{|c|}{ Muestreo } \\
\hline & Ago & Oct & Dic & Feb & $\mathrm{Abr}$ & Jun \\
\hline Alphestes immaculatus & $\mathrm{P}$ & & & & & \\
\hline Hyporhamphus unifasciatus & $\mathrm{P}$ & & & & & \\
\hline Atherinella eriarcha & $\mathrm{P}$ & & & & & \\
\hline Tylosurus fodiator & $\mathrm{P}$ & & & & & \\
\hline Rypticus nigripinnis & $\mathrm{P}$ & & & & & \\
\hline Trachinotus rhodopus & $\mathrm{P}$ & & & & & \\
\hline Scomberomorus sierra & $\mathrm{P}$ & $\mathrm{P}$ & & & & \\
\hline Pomadasys branickii & $\mathrm{P}$ & $\mathrm{P}$ & $\mathrm{P}$ & & $\mathrm{P}$ & $\mathrm{P}$ \\
\hline Arothron meleagris & & $\mathrm{P}$ & & & & \\
\hline Carcharhinus leucas & & $\mathrm{P}$ & & & & \\
\hline Diodon holocanthus & $\mathrm{P}$ & & $\mathrm{P}$ & & & \\
\hline Eleotris picta & & $\mathrm{P}$ & & & & \\
\hline Chanos chanos & $\mathrm{P}$ & $\mathrm{P}$ & $\mathrm{P}$ & $\mathrm{P}$ & & \\
\hline Diapterus peruvianus & $\mathrm{P}$ & $\mathrm{P}$ & $\mathrm{P}$ & $\mathrm{P}$ & $\mathrm{P}$ & \\
\hline Eucinostomus currani & $\mathrm{P}$ & $\mathrm{P}$ & $\mathrm{P}$ & $\mathrm{P}$ & $P$ & \\
\hline Oxyzygonectes dovii & $\mathrm{P}$ & $\mathrm{P}$ & $\mathrm{P}$ & $\mathrm{P}$ & $\mathrm{P}$ & $\mathrm{P}$ \\
\hline Sphoeroides annulatus & $\mathrm{P}$ & $\mathrm{P}$ & $\mathrm{P}$ & $\mathrm{P}$ & $\mathrm{P}$ & $\mathrm{P}$ \\
\hline Poeciliopsis elongata & $\mathrm{P}$ & $\mathrm{P}$ & $\mathrm{P}$ & $\mathrm{P}$ & $\mathrm{P}$ & $\mathrm{P}$ \\
\hline Lile stolifera & $\mathrm{P}$ & $\mathrm{P}$ & $\mathrm{P}$ & $\mathrm{P}$ & $\mathrm{P}$ & $\mathrm{P}$ \\
\hline Lutjanus colorado & $\mathrm{P}$ & $\mathrm{P}$ & $\mathrm{P}$ & $\mathrm{P}$ & $\mathrm{P}$ & $\mathrm{P}$ \\
\hline Kyphosus elegans & $\mathrm{P}$ & $\mathrm{P}$ & & & & $\mathrm{P}$ \\
\hline Mugil cephalus & $\mathrm{P}$ & $\mathrm{P}$ & $\mathrm{P}$ & & & $\mathrm{P}$ \\
\hline Ctenogobius sagittula & $\mathrm{P}$ & $\mathrm{P}$ & $\mathrm{P}$ & $\mathrm{P}$ & & $\mathrm{P}$ \\
\hline Centropomus armatus & $\mathrm{P}$ & $P$ & $\mathrm{P}$ & $\mathrm{P}$ & & $\mathrm{P}$ \\
\hline Haemulopsis leuciscus & $\mathrm{P}$ & $\mathrm{P}$ & & $\mathrm{P}$ & $\mathrm{P}$ & \\
\hline Caranx caninus & $\mathrm{P}$ & $\mathrm{P}$ & & $\mathrm{P}$ & & $\mathrm{P}$ \\
\hline Atherinella argentea & $\mathrm{P}$ & $\mathrm{P}$ & & $\mathrm{P}$ & & $\mathrm{P}$ \\
\hline Mugil curema & & $\mathrm{P}$ & $\mathrm{P}$ & $\mathrm{P}$ & $\mathrm{P}$ & \\
\hline Haemulopsis elongatus & & $\mathrm{P}$ & $\mathrm{P}$ & & $\mathrm{P}$ & $\mathrm{P}$ \\
\hline Elops affinis & & $\mathrm{P}$ & & & & $\mathrm{P}$ \\
\hline
\end{tabular}

\begin{tabular}{|c|c|c|c|c|c|}
\hline \multirow{2}{*}{ Especie } & \multicolumn{5}{|c|}{ Muestreo } \\
\hline & Ago Oct & Dic & Feb & Abr & Jun \\
\hline Lutjanus argentiventris & & $\mathrm{P}$ & $\mathrm{P}$ & $\mathrm{P}$ & \\
\hline Eucinostomus gracilis & & $\mathrm{P}$ & $P$ & $\mathrm{P}$ & \\
\hline Lutjanus novemfasciatus & & $\mathrm{P}$ & & $\mathrm{P}$ & \\
\hline Atherinella pachylepis & & $\mathrm{P}$ & & $\mathrm{P}$ & \\
\hline Caranx vinctus & & $\mathrm{P}$ & & $\mathrm{P}$ & $\mathrm{P}$ \\
\hline Centropomus medius & & $\mathrm{P}$ & & & $\mathrm{P}$ \\
\hline Caranx caballus & & $\mathrm{P}$ & & & \\
\hline Atherinella panamensis & & $\mathrm{P}$ & & & \\
\hline Oligoplites saurus & & $\mathrm{P}$ & $\mathrm{P}$ & & \\
\hline Centropomus robalito & & $\mathrm{P}$ & & & \\
\hline Alphestes multiguttatus & & & $\mathrm{P}$ & & \\
\hline Abudefduf troschelii & & & $\mathrm{P}$ & & \\
\hline Lutjanus aratus & & & $\mathrm{P}$ & & \\
\hline Oligoplites altus & & & $\mathrm{P}$ & & \\
\hline Centropomus viridis & & & $\mathrm{P}$ & $\mathrm{P}$ & \\
\hline Chaetodon humeralis & & & & $\mathrm{P}$ & \\
\hline Lutjanus guttatus & & & & $\mathrm{P}$ & \\
\hline Lutjanus jordani & & & & $\mathrm{P}$ & \\
\hline Etropus crossotus & & & & $\mathrm{P}$ & \\
\hline Stegastes leucorus & & & & $\mathrm{P}$ & \\
\hline Gymnothorax dovii & & & & $\mathrm{P}$ & \\
\hline Gerres cinereus & & & & $\mathrm{P}$ & $\mathrm{P}$ \\
\hline Anchoa spinifer & & & & & $\mathrm{P}$ \\
\hline Centropomus nigrescens & & & & & $\mathrm{P}$ \\
\hline Chloroscombrus orqueta & & & & & $\mathrm{P}$ \\
\hline Lutjanus peru & & & & & $\mathrm{P}$ \\
\hline Peprilus ovatus & & & & & $\mathrm{P}$ \\
\hline Pomadasys nitidus & & & & & $\mathrm{P}$ \\
\hline Selene brevoortii & & & & & $\mathrm{P}$ \\
\hline
\end{tabular}




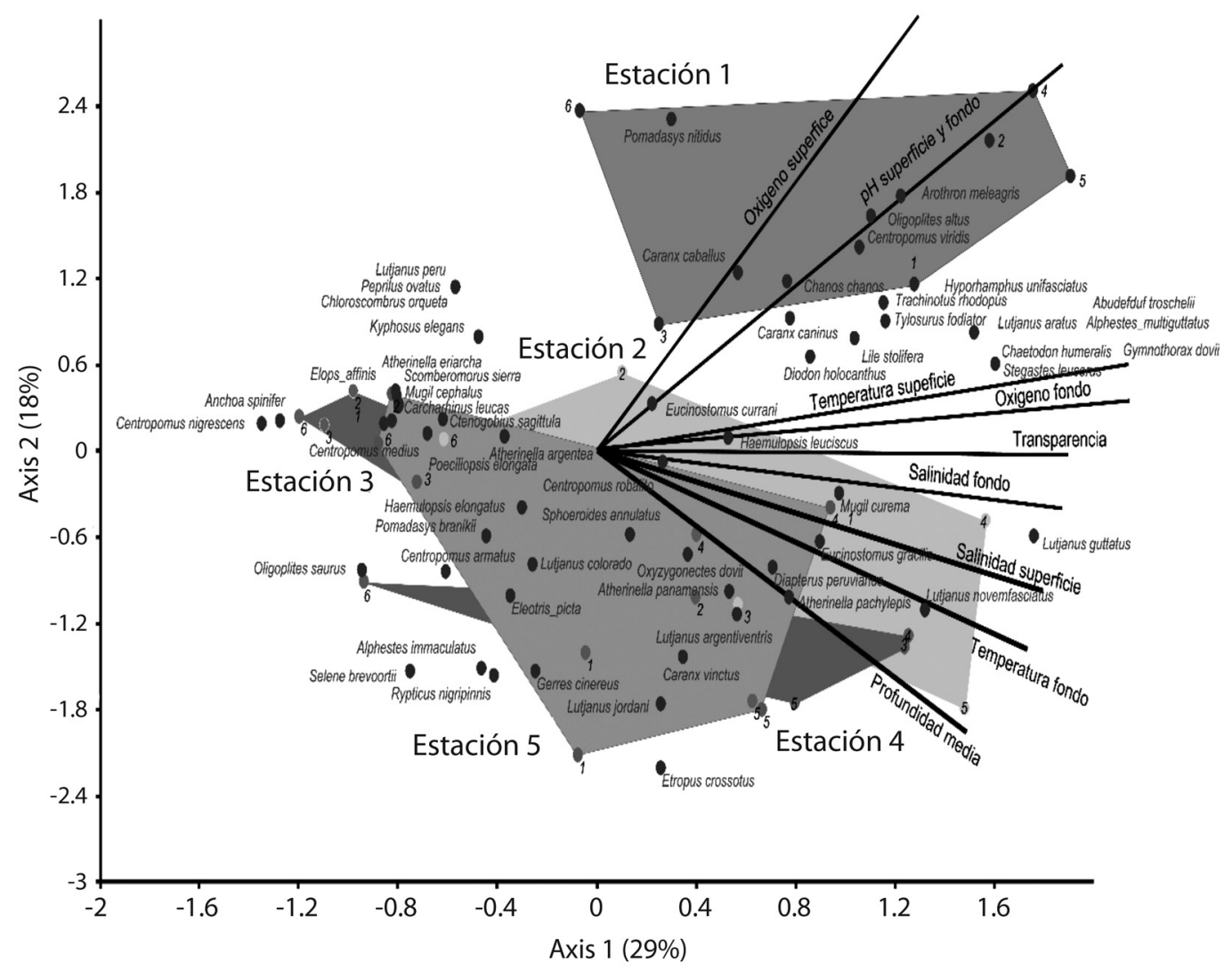

Fig. 2. Análisis de correspondencias canónicas para las estaciones y muestreos, según la composición de especies y las variables ambientales del estero. 1 al $6=$ meses de muestreo. Cada área en color enmarca cada uno de los eventos de muestreo según estación de la 1 (boca) a la 5 (interior) del estuario.

Fig. 2. Canonical Correspondence Analysis of the stations and sampling events, based in the composition of species and environmental variables in the estuary. 1 to $6=$ months of sampling. Each color area contained the sampling events by station, from 1 (mouth) to the 5 (inner of the estuary).

ubicadas en el extremo opuesto como el caso del róbalo (Centropomus nigrescens) y la sardinita (Anchoa spinifer) (Fig., 2). En ese sentido, de la estación cinco y dos se sigue un patrón de incremento de los valores de dichas variables hacia la boca del estuario (Fig. 2). En esa misma dirección sobre el eje 1 , se ve el cambio en la abundancia de las especies de estuarinas a más oceánicas (Fig. 2).

Las variables ambientales que mejor explican los cambios en la composición de la ictiofauna en el estero, fueron la transparencia en conjunto con el $\mathrm{pH}$ y el oxígeno con un valor de asociación de $29 \%$ (BIOENV; $\mathrm{p}=0.003)$.

\section{DISCUSIÓN}

Comportamiento de las variables físico químicas: Coincidiendo con lo reportado por Castillo-Rivera, Zárate, \& Sanvicente-Añorve (2003), la baja profundidad media aunada a la escasa longitud del estero contribuyó a que las variables físico químicas variaran con menor grado en el espacio respecto al tiempo. Espacialmente, las variables mostraron condiciones predominantemente marinas en las estaciones uno y dos (las más externas en el estuario), con mayor transparencia, salinidad, $\mathrm{pH}$ y oxígeno disuelto que las estaciones internas $(3,4$ y 5$)$, 
en donde el $\mathrm{pH}$ y la salinidad disminuyeron por la mezcla con el agua dulce aportada por los ríos y mayor cantidad de materia orgánica en descomposición, en cuyo proceso se presenta el consumo de oxigeno por oxidación y se genera ácido sulfhídrico que contribuye con la disminución del pH del agua (Burd, Nemec, \& Brinkhurst, 1990; Hernández, Aguilar-Betancourt, \& González-Sansón, 2009). La similitud espacial de la temperatura, se relaciona con la poca longitud, profundidad y al alta dinámica de flujo y reflujo, ya que en la costa cercana se alcanzan mareas de hasta $3.2 \mathrm{~m}$. Ibarra-Obando $\&$ Poumian-Tapia (1991) determinaron que en ecosistemas similares hasta el $60 \%$ del agua puede ser evacuada en un ciclo de mareas.

Temporalmente, la mayor concentración de oxígeno y $\mathrm{pH}$ se presentó en la época seca, similar a lo reportado por VelázquezVelázquez, Vega-Cendejas, \& Navarro-Alberto (2008). Estos valores se correlacionan con el aumento de la transparencia del agua producto de la ausencia de precipitación. En el caso de la temperatura, los bajos valores obtenidos en setiembre y octubre se explican por ser los meses de mayor precipitación $\mathrm{y}$, en diciembre y enero, por la influencia de los primeros frentes fríos del norte, en abril se registraron las temperaturas más altas del año para la zona de estudio (Instituto Meteorológico Nacional de Costa Rica, 2011). Estos patrones de estacionalidad han sido encontrados en otros ecosistemas estuarinos del país, como en el Golfo de Nicoya (Epifanio, Maurer, \& Dittel, 1983) y el humedal Térraba-Sierpe (Picado, 2015).

\section{Estructura ictiofaunística y variación} espacio temporal: La abundancia total corresponde a condiciones de un estuario tropical menos abundante y de mayor cantidad de especies, similar a la obtenida por Feutry et al. (2010), con 1591 individuos en el estero de Zancudo, Golfo Dulce, Costa Rica. La abundancia fue superior en octubre, mes de mayor registro de precipitación de la zona, similar a lo encontrado por Phillips et al. (1983) en Punta Morales, Costa Rica. El aumento de las lluvias incrementa el flujo de materia orgánica alóctona y nutrientes hacia el sistema, estimulando las cadenas tróficas que ingresen al sistema a alimentarse (Castillo -Rivera et al., 2003).

Se infiere que la estación tres presentó mayor cantidad de individuos y mayor riqueza debido a que este sitió presentó valores intermedios de salinidad y mayor variedad de hábitats (fondos de arena, piedra, lodo, troncos, hojas sumergidas, raíces de mangle). Freitas, Petrere, \& Barrella (2005) e Ibarra et al. (2005), encontraron que en donde la heterogeneidad espacial es mayor, se proporcionan sitios ideales para una mayor abundancia y diversidad de especies. Las familias Poecilidae, Mugilidae y Clupeidae son ictiofauna importante en los estuarios (Sandoval-Huerta et al., 2014; Bharadhirajan, Murugan, Gopalakershanan, \& Murugesan, 2015). Con esta investigación es posible explicar aspectos de la abundancia debida a las características fisiológicas y metabólicas que les permiten adaptarse, siendo en su mayoría, especies eurihalinas y euritermas con gran capacidad de osmoregulación y resistencia a niveles bajos de oxígeno y cambios de $\mathrm{pH}$. Por ejemplo, Poeciliopsis elongata, la especie más frecuente en las capturas, posee una amplia tolerancia a fluctuaciones dea temperatura, salinidad y oxígeno disuelto (Espinoza, 2007).

En comparación con otros estuarios de la Costa Pacífica de Costa Rica, la riqueza es similar a la reportada por Araya (1988), en los esteros Damas y Palo Seco en Quepos y Parrita, con 66 especies y 30 familias, y levemente inferior a las especies reportadas por Rojas et al. (1994) en el Golfo de Nicoya con 75 especies de 25 familias, y Feutry et al. (2010) en el manglar de Zancudo en el Golfo Dulce, con 82 especies de 30 familias. Se infiere que esta similitud se explica por el remplazo estacional equitativo de especies sucedido en este trabajo y también descrito por Ayala-Pérez, RamosMiranda, \& Flores-Hernández (2003) y Castillo -Rivera et al. (2003), en donde se apreció de forma general que un grupo de especies fue muestreado al inicio del periodo y otro al final, mientras que otras se distribuyeron a lo largo de todo periodo de estudio (Cuadro 4). 
Las raíces de Rhizophora mangle propiciaban patrones de conducta de protección en los peces, principalmente juveniles. La importancia de esta protección radica en que esta familia hace un fuerte aporte dentro de los recursos marinos costeros de Costa Rica, sosteniendo las pesquerías de algunas zonas del país (Soto-Rojas, Mejía-Arana, Palacios, \& Hiramatsu, 2009).

El estero, como ecosistema esencial para los peces, presentó un alto índice de diversidad. Los valores obtenidos son similares a los registrados para esteros del Pacífico Costarricense (León, 1973; Araya, 1988). La mayor diversidad de la estación dos se relaciona a la existencia de un tramo de sustrato sólido, con gran cantidad de nichos ideales para la protección de muchas especies, aumentando así el índice de diversidad. Esto concuerda con estudios afines, donde se ha determinado que la multiplicidad de fondos influye en la composición íctica (de Grado, Bashirullah, \& Prieto, 2000; Benítez et al., 2007). La disponibilidad de refugios en esta estación explica la protección contra depredadores, lo que fortalece las tasas de crecimiento y reduce la mortalidad (Rozas \& Odum, 1988; Lubbers, Boynton, \& Kemp, 1990; Humphries, Potter, \& Loneragan, 1992). Las especies de la familia Haemulidae (roncadores) son las que más contribuyen al índice, en esta estación. Allen \& Robertson (1998) reportan que esta familia, habita los esteros y está compuesta por peces de pequeños que pueden utilizar los intersticios de las zonas rocosas como refugio.

El índice de equidad para el estero de Tamarindo, fue similar al obtenido por Araya (1988) para los esteros de Damas y Palo Seco de Costa Rica (0.6). En este estudio el valor de equidad es similar a lo descrito por López (2005), por lo tanto, se considera que la distribución de los patrones de abundancia es similar entre las localidades, lo que permite una dominancia baja, sugiriendo cierta estabilidad y homogeneidad de la comunidad de peces en el área de estudio. La estación tres mostró una menor distribución de la abundancia entre las especies, tanto espacial como temporalmente. La variabilidad en la composición de las especies dominantes puede ser consecuencia de diferencias en la salinidad (Sandoval-Huerta et al., 2014), lo que se relaciona a la mayor equidad de la estación cinco (ramal interno) restringiendo el hábitat a un grupo de especies poco abundantes con capacidad eurihalina, donde ninguna especie presentó dominancia. En concordancia con López (2005) y Espinoza (2007) se han registrado otras investigaciones con una mayor presencia íctica hacia el final del estuario, por ser ahí donde la carga de contenido orgánico se acumula; además, sistemas con mayor heterogeneidad espacial pueden proporcionar una mayor disponibilidad de micro-ambientes, favoreciendo a su vez, a una mayor riqueza y colonización de especies.

Como explican Caballero-Vásquez, Gamboa-Pérez, \& Schmitter-Soto (2005), la influencia marina tiene implicación en la composición de especies que van a pertenecer en menor o mayor grado al componente dulceacuícola, marino o estuarino. La semejanza íctica entre las estaciones tres y cinco (Fig. 1), se atribuye a la influencia directa que tienen de los ríos Matapalo y San Andrés, los cuales propician valores bajos de salinidad y $\mathrm{pH}$, que se reflejan en una ictiofauna típica dulceacuícola. En este tipo de sistemas la velocidad máxima de la corriente de marea se da en la boca y disminuye hacia los ramales internos, resultados semejantes han sido publicados por Ibarra-Obando \& Poumian-Tapia (1991) y Ortiz, Huerta-Tamayo, \& Hinojosa (2003).

Según los resultados de la sucesión de especies, se infiere que el estero de Tamarindo presenta grupos definidos de especies según la época del año, investigaciones llevadas a cabo por Castillo-Rivera, Ortiz-Burgos, \& Zárate-Hernández (2011) han obtenido resultados similares, con una sucesión cíclica dentro del año. Un grupo que se presenta en época lluviosa, otro en época seca, y un grupo que permanece constantemente en el año y que podría considerarse como los residentes del estero (Cuadro 5). Es común que las comunidades de peces presenten diferencias estacionales en la composición de especies, los cuales son explicados por Wootton (1990), Callow \& Petts 
(1994) y Castillo-Rivera et al. (2011), como resultado de los desfases en los tiempos de desove y las correspondientes inmigraciones y emigraciones secuenciales de las especies, así como por las fluctuaciones estacionales en la disponibilidad de alimento, que a su vez dependen de las condiciones climáticas que imperen en cada época del año. Así, una sucesión estacional de especies puede reflejar las diferentes respuestas de estas a las condiciones físicas ambientales (Castillo-Rivera et al., 2011).

\section{Relación de la ictiofauna con las varia-} bles ambientales: La influencia de las variables ambientales, tales como la salinidad, profundidad, turbidez, $\mathrm{pH}$ y oxígeno disuelto, sobre la diversidad de peces, ha sido mencionada en varios estudios (Kushlan, 1976; Cyrus \& Blaber, 1992; Thiel, Sepulveda, Kafemann, \& Nellen, 1995; Fraser, 1997). En el caso del oxígeno del estero de Tamarindo, similar a lo reportado por Velázquez-Velázquez et al. (2008), se corrobora que es el parámetro más heterogéneo, que conduce a importantes cambios de la composición íctica entre cada sitio muestreado. Las disminuciones en la composición íctica, obtenidas en este estudio podrían estar asociadas a los materiales en suspensión (Hernández, 1995) y contaminación antrópica (Aston, 1980; Clark, 2001) acarreada por los ríos que irrigan el estuario.

Se puede concluir que la comunidad de peces del estero de Tamarindo es rica en especies, diversa y equitativa, en donde se presentan procesos de reclutamiento de varias de las especies más importantes para el sector pesquero de la costa pacífica. No obstante, por su poca longitud y baja profundidad, es un ecosistema frágil, propenso a contaminación por agricultura y urbanización turística, con implicaciones negativas sobre la comunidad íctica.

\section{AGRADECIMIENTOS}

Por los aportes a esta investigación se agradece a José Walter López López de la Asociación APROTORBA, al director del Parque Nacional Marino Las Baulas, Rodney Piedra
Chacón, al Consejo Nacional para Investigaciones Científicas y Tecnológicas (CONICIT) del Ministerio de Ciencia y Tecnología, a la Fundación The Leatherback Trust, a la Presidenta de la Fundación Parque Marino del Pacífico, Juana María Coto Campos y al Director Ejecutivo, Ángel Herrera Ulloa, por facilitar equipo de medición para los trabajos de campo.

\section{RESUMEN}

Las comunidades de peces en manglares tienen una importancia ecológica y socio-económica. El manejo de este tipo de hábitat está basado en datos sobre sus patrones espaciales y temporales. El objetivo de este estudio es determinar la estructura espacio temporal de la comunidad íctica del estero de Tamarindo, Costa Rica. Se efectuaron seis muestreos, entre agosto del 2009 y junio del 2010, en cinco estaciones; usando cuatro artes de pesca. Se registró la temperatura, oxígeno, salinidad, $\mathrm{pH}$ y transparencia del agua en cada estación. Se recolectaron 1357 individuos distribuidos en 28 familias y 59 especies. Las familias con mayor número de especies son Carangidae y Lutjanidae, y las más abundantes en términos de frecuencia de captura son Poecilidae, Mugilidae y Clupeidae, sobresaliendo la sardina Poeciliopsis elongata. Temporalmente, la diversidad alcanzó un mínimo de1.55 en diciembre y un máximo de 1.96 en abril; la riqueza un mínimo de 22 especies en febrero y un máximo de 25 en diciembre. Espacialmente, la diversidad alcanzó un mínimo de 1.96 en la boca del estuario y un máximo de 2.79 en la región media del estuario; la riqueza con un mínimo de 24 especies se dio en la parte interna y obtuvo un máximo de 30 en la parte media del estuario. Se determinó un patrón temporal de sucesión de especies. La comunidad íctica presenta un patrón de homogenización en la similitud desde la boca hacia la parte interna del estuario. En la parte interna la abundancia de peces es menor, pero la riqueza, diversidad y equitatividad es mayor. Finalmente, procesos de reclutamiento de varias especies importantes para el sector pesquero se dan en este estuario, haciéndolo frágil y propenso a contaminación e impactos antropogénicos, debido a su poca longitud y baja profundidad.

Palabras clave: Índices de diversidad, estero Tamarindo, riqueza de especies, patrones de distribución espacio temporal, estructura íctica.

\section{REFERENCIAS}

Allen, G., \& Robertson, R. (1998). Peces del Pacifico Oriental Tropical. Bathurst, Australia: Crawford House Press Pty Ltd. 
Alpírez, O. (1985). Ictiofauna de la vertiente Pacífica de Costa Rica. Brenesia, 24, 297-318.

Araya, H. (1988). Diversidad, distribución, abundancia y relaciones tróficas de peces en estero Damas y estero Palo Seco. Aguirre (Quepos), Parrita, Puntarenas. Costa Rica (Tesis de Licenciatura). San José, Costa Rica: Universidad de Costa Rica.

Aston, S. (1980). Nutrients, dissolved gases and general biogeochemistry in estuaries. Hoboken, NJ: Wiley.

Ayala-Pérez, L. A., Ramos-Miranda, J., \& Flores-Hernández, D. (2003). La comunidad de peces de la Laguna de Términos: estructura actual comparada. Revista de Biología Tropical, 51(3-4), 783-793.

Bejarano, I., Blanco, M. P., \& Mojica, J. I. (2006). La comunidad ictica del rio Mesay durante el periodo de aguas altas (Caquetá, Amazonia colombiana). Caldasia, 28(2), 359-370.

Benítez, C., Ruiz,, J., Marcial, E., Blanco, M., López, C., López, P., \& Castañeda, A. (2007). Diversidad y abundancia de la comunidad de peces del estero "El Custodio", Municipio de Compostela, Nayarit, México. Revista electrónica de Veterinaria, 8, 1-13.

Bharadhirajan, P., Murugan, S., Gopalakershanan, A., \& Murugesan, P. (2015). Finfish diversity in Coleroon estuary, Southeast coast of India. Indian Journal of Marine Sciences, 44, 104-109.

Burcham, J. (1988). Fish communities and environmental characteristics of two lowland streams in Costa Rica. Revista de Biología Tropical, 36, 273-285.

Burd, B. J., Nemec, A., \& Brinkhurst, R. O. (1990). The development and application of analytical methods in benthic marine infaunal studies. Advances in Marine Biology, 26, 169-247.

Bussing, W., \& López, M. (1977). Distribución y aspectos ecológicos de los peces de las cuencas hidrográficas de Arenal, Bebedero y Tempisque, Costa Rica. Revista de Biología Tropical, 25, 13-37.

Bussing, W., \& López, M. (1994). Peces demersales y pelágicos costeros del Pacífico de Centro América meridional. Revista de Biología Tropical, (Publicación Especial), 1-164.

Caballero-Vázquez, J. A., Gamboa-Pérez, H. C., \& Schmitter-Soto, J. J. (2005). Composición y variación espacio-temporal de la comunidad de peces en el Sistema Lagunar Chacmochuch, Quintana Roo, México. Hidrobiológica, 15(2), 215-225.

Callow, P., \& Petts, G. (1994). The river handbook: hydrological and ecological principles. Oxford, United Kingdom: Blackwell Science.

Carrasco, J., \& Soriguer, M. (2015). Distribution and diversity of fish communities in the continuous longitudinal of Río Plátano, Honduras. Mesoamericana, 19, 9-21.

Castillo-Rivera, M., Zárate, R., \& Sanvicente-Añorve, L. (2003). Patrones de la diversidad de peces en la laguna de Pueblo Viejo, Veracruz, México. Hidrobiológica, 13(4), 289-298.

Castillo-Rivera, M., Ortiz-Burgos, S., \& Zárate-Hernández, R. (2011). Estructura de la comunidad de peces estuarinos en un hábitat con vegetación sumergida: variación estacional y nictémera. Hidrobiológica, 21(3), 311-321.

Chicas, F. (2001). Peces juveniles en una poza de marea, Reserva Forestal Térraba-Sierpe, Puntarenas, Costa Rica. Revista de Biología Tropical, 49(Supl. 2), 307-314.

Clark, R., (2001). Marine Pollution. Nueva York: Oxford University Press.

Clarke, K., \& Warwick, R. (1994). Change in marine communities: an approach to statistical analysis and interpretation. Bournemouth, UK: Bourne.

Constanz, G. D., Bussing, W. A., \& Saul, W. G. (1981). Freshwaler fishes of Corcovado National Park. Costa Rica. Proceedings of the Academy of Natural Sciences of Philadelphia, 133, 15-9.

Crawley, M. J. (2007). The R Book. Chichester, UK: John Wiley \& Sons.

Cyrus, D. P., \& Blaber, S. J. M. (1992). Turbidity and salinity in a tropical northern Australian estuary and their influence on fish distribution. Estuarine, Coastal and Shelf Science, 35(6), 545-563.

de Grado, A. A., Bashirullah, A., \& Prieto, A. (2000). Variación espacio-temporal de la comunidad de peces en Laguna Grande de Obispo, Golfo de Cariaco, Estado Sucre, Venezuela. Acta Científica Venezolana, 51(2), 96-103.

De Marco, S., Pan, J., Bassini, S., \& Vallina, M. (2013). Biodiversidad en estuarios. En J. Marcovecchio \& R. Freije (Eds.). Procesos químicos en estuarios (pp. 109-120). Bahía Blanca, Argentina: Editorial de la Universidad Tecnológica Nacional.

Epifanio, C. E., Maurer, D. \& Dittel, A. I. (1983). Seasonal changes in nutrients and dissolved oxygen in the Gulf of Nicoya, a tropical estuary on the Pacific coast of Central America. Hydrobiologia, 101(3), 231-238.

Espinoza, M. (2007). Composición y estructura de las comunidades de peces del río La Cañaza, Pacífico Sur de Costa Rica. Brenesia, 67, 35-43.

Feutry, P., Hartmann, H. J., Casabonnet, H., \& Umaña, G. (2010). Preliminary analysis of the fish species of the Pacific Central American mangrove of Zancudo, 
Golfo Dulce, Costa Rica. Wetlands ecology and management, 18(6), 637-650.

Fisher, W., Krupp, F., Schneider, W., Sommer, C., Carpenter, K., \& Niem, V. (1995). Guía FAO para la identificación de especies para los fines de la pesca. Pacifico Centro-Oriental. Organización de las Naciones Unidas para la Agricultura y la Alimentación, Roma, Italia: FAO.

Fraser, T. H. (1997). Abundance, seasonality, community indices, trends and relationships with physicochemical factors of trawled fish in upper Charlotte Harbor, Florida. Bulletin of Marine Science, 60(3), 739-763.

Freitas, C. E. C., Petrere, M., \& Barrella, W. (2005). Natural and artificially-induced habitat complexity and freshwater fish species composition. Fisheries Management and Ecology, 12(1), 63-67.

Hammer, Ø., Harper, D. A. T., \& Ryan, P. D. (2001). PAST: Palontological Satatistics software package for education and data analysis. Paleontologia Electronica, $4,1-9$.

Hernández, A., (1995). Régimen hidrográfico del estero Real (Nicaragua) y sus variaciones estacionales. En J. Zamarro (Ed.). Actas del Simposium. Ecosistema de manglares en el Pacífico Centroamericano y su recurso de Post-Larvas de camarones peneidos. (pp. 48-60). San Salvador, El Salvador: Programa Regional de Apoyo al Desarrollo de la Pesca del Istmo Centroamericano.

Hernández, I., Aguilar Betancourt, C., \& González Sansón, G. (2009). Variaciones en la abundancia de peces en sitios con diferente grado de contaminación del sublitoral de Ciudad de La Habana, Cuba. Revista de Biología Tropical, 57(4), 977-992.

Humphries, P., Potter, I. C., \& Loneragan, N. R. (1992). The fish community in the shallows of a temperate australian estuary: Relationships with the aquatic macrophyte Ruppia megacarpaand environmental variables. Estuarine, Coastal and Shelf Science, 34(4), 325-346.

Ibarra-Obando, S. E., \& Poumian-Tapia, M. (1991). The effect of tidal exclusion on salt marsh vegetation in Baja California, Mexico. Wetlands Ecology and Management, 1(3), 131-148.

Ibarra, A. A., Park, Y. S., Brosse, S., Reyjol, Y., Lim, P., \& Lek, S. (2005). Nested patterns of spatial diversity revealed for fish assemblages in a west European river. Ecology of Freshwater Fish, 14(3), 233-242.

Instituto Meteorológico Nacional de Costa Rica. (2011). Clima en Costa Rica Pacífico Norte. Instituto Meteorológico Nacional, San José, Costa Rica. Recuperado de www.imn.ac.cr/educacion/climacr/pacifico_norte. htm
Jiménez, J., (1999). Ambiente, distribución y características estructurales en los manglares del Pacífico de Centro América: Contrastes climáticos En A. YáñezArancibia \& A. Lara-Domínguez (Eds.). Ecosistemas de Manglar en América Tropical (pp. 51-70). Veracruz, México: Instituto de Ecología A.C.

Krebs, C. J., (1999). Ecological methodology. California, EEUU: Benjamin/Cummings.

Kushlan, J. A. (1976). Environmental stability and fish community diversity. Ecology, 57(4), 821-825.

León, P. E. (1973). Ecología de la ictiofauna del Golfo de Nicoya, Costa Rica, un estuario tropical. Revista de Biología Tropical, 21(1), 5-30.

López, L. D. C., (2005). Estructura de la comunidad íctica en el estero de Rancho Bueno, Baja California Sur, México (Tesis Doctoral). La Paz, México: Instituto Politécnico Nacional-Centro Interdisciplinario de Ciencias Marinas.

Lubbers, L., Boynton, W., \& Kemp, W. (1990). Variations in structure of estuarine fish communities in relation to abundance of submersed vascular plants. Marine Ecology Progress Series, 65, 1-14.

Martínez, A., (2015). Análisis de ADN ambiental en estuarios productores de moluscos (Tesis de Maestría). Oviedo, España: Universidad de Oviedo.

Nelson, J., (2006). Fishes of the World. New Jersey, EEUU: Wiley.

Ortiz, M., Huerta-Tamayo, L., \& Hinojosa, A. (2003). Transporte de sedimento por tracción de marea en el estero de Punta Banda, Baja California, México. GEOS, 23(3), 283-294.

Phillips, P. C., Astorga, I., Hidalgo, C., \& Villarreal, A. (1983). Diel and monthly variation in abundance, diversity and composition of littoral fish populations in the Gulf of Nicoya, Costa Rica. Revista de Biología Tropical, 31(2), 297-306.

Picado, J. (2015). Spatial and temporal salinity distribution in the water column of the estuarine sector of the Térraba Sierpe National Wetland, Costa Rica. Revista de Biología Tropical, 63(1), 75-96.

Quinn, G. P., \& Keough, M. J. (2002). Experimental design and data analysis for biologists. Cambridge, UK: Cambridge University Press.

Rojas, J., Pizarro, F., \& Castro, M. (1994). Diversidad y abundancia íctica en tres áreas de manglar en el Golfo de Nicoya, Costa Rica. Revista de Biología Tropical, 42, 663-672.

Rozas, L. P., \& Odum, W. E. (1988). Occupation of submerged aquatic vegetation by fishes: testing the roles of food and refuge. Oecologia, 77(1), 101-106. 
Sandoval-Huerta, E. R., Madrigal-Guridi, X., EscaleraVázquez, L. H., Medina-Nava, M., \& DomínguezDomínguez, O. (2014). Estructura de la comunidad de peces en cuatro estuarios del Pacífico mexicano central. Revista mexicana de biodiversidad, 85(4), 1184-1196.

Soto-Rojas, R. L., Mejía-Arana, F., Palacios, J. A., \& Hiramatsu, K. (2009). Reproducción y crecimiento del pargo mancha Lutjanus guttatus (Pisces: Lutjanidae) en el Golfo de Nicoya, Costa Rica. Revista de Biología Tropical, 57(1-2), 125-131.

Thiel, R., Sepulveda, A., Kafemann, R., \& Nellen, W. (1995). Environmental factors as forces structuring the fish community of the Elbe Estuary. Journal of Fish Biology, 46(1), 47-69.

Tiffer, R., Mata, A., Losilla, M., Cervantes, S., Cajiao, M., Damson, M., Araúz, R., \& Marín, M. (2003). Diagnóstico Ambiental del Parque Nacional Marino Las
Baulas de Guanacaste. Plan de Manejo del Parque Nacional Marino Las Baulas de Guanacaste - ACT. San José, Costa Rica: Centro Científico Tropical.

Velázquez-Velázquez, E., Vega-Cendejas, M. E., \& Navarro-Alberto, J. (2008). Spatial and temporal variation of fish assemblages in a coastal lagoon of the Biosphere Reserve La Encrucijada, Chiapas, Mexico. Revista de Biología Tropical, 56(2), 557-574.

Winemiller. K. O. (1983). An introduction to the freshwater fish communities of Corcovado National Park.Costa Rica. Brenesia, 211, 47-66.

Winemiller, K., \& Morales, N. (1989). Comunidades de peces del Parque Nacional Corcovado luego del cese de las actividades mineras. Brenesia, 31, 75-91.

Wootton, R. (1990). Ecology of teleost fishes. Nueva York: Chapman \& Hall. 